\title{
How I Learned to Love the Bomb: Defcon and the Ethics of Computer Games
}

\author{
Miguel Sicart \\ Assistant Professor \\ Center for Computer Game Research \\ IT University of Copenhagen \\ http: / /www.itu.dk \\ Junior Research Associate \\ IEG - Oxford University \\ http: //web.comlab.ox.ac.uk/oucl/research/areas/ieg/ \\ GPI - University of Hertfordshire \\ http://philosophyofinformation. net/centre/gpi/index.html \\ miguel@itu.dk \\ www.miguelsicart.net
}

\begin{abstract}
In this paper I present an analysis of the ontology and ethics of computer games from an Information Ethics perspective. This analysis uses the concepts of Level of Abstraction and Gradient of Abstraction, as defined by Luciano Floridi's Information Ethics, applied to the specific study of computer games. The goal of this paper is to argue for the consideration of games as interesting ethical objects and experiences. Computer games appeal to a player capable of ethical reasoning in her interaction with simulated environments and rule systems. This paper provides a theoretical model for the study of the ethics of computer games both as designed objects, and as player experiences.
\end{abstract}

Keywords: Information Ethics, Computer Game Ethics, Method of Abstraction.

\section{Introduction}

Computer games are the dominant paradigm of $21^{\text {st }}$ Century entertainment. Their worldwide economic success inspires the technological research that extends the boundaries of game-related computation. But computer games have too a reputation of being intrinsically unethical forms of entertainment that corrupt the values of society by promoting the wrong personal and social virtues. ${ }^{1}$ This reputation is caused and exemplified by the simulation of violence that many computer games use as a vehicle for the expression of conflict. ${ }^{2}$ There is an ethical tension between the perception of computer games as infantile entertainment, and the demands to the game industry from their core adult audience.

In any case of moral concerns it is necessary to question the ethical origins of this perception. This paper argues for an interpretation of the ethics of games as designed

1 Carnagey et al. (2006).

2 Juul (2005), pp. 92 - 116, and Salen and Zimmerman (2004), pp. 74-78 \& 254-255. 
software systems that are experienced by moral agents. The goal of this paper is to present a model for the ethical analysis of games. This model will use concepts from Luciano Floridi's Information Ethics ${ }^{3}$, applied to the analysis of the independent multiplayer computer game Defcon (Introversion Software: 2006).

This is a paper on Information Ethics. As such, it applies the theoretical framework proposed by Floridi to the analysis of computer game. The terminology, and the Method of Abstraction are direct appropriations from Information Ethics, but their application to computer games is entirely original. Furthermore, concepts like Procedural and Semantic Gradients of Abstraction, are original additions to Information Ethics theory, first presented in this paper.

Information Ethics provides a comprehensive framework for the analysis of computer games for three fundamental reasons: first, the focus on information as an ontology facilitates the analysis of computer games as systems, beyond their audiovisual and aural components, yet integrating these in the whole meaning of the game experience; second, Information Ethics provides a strong vocabulary, with roots in Information Theory and Computer Science, which affords precise definitions of systems, and their interaction modes, from an ethical perspective; third, Information Ethics is a constructivist theory with a strong anthropological model ${ }^{4}$, which in turn can be used to outline a model of ethical player. In any case, this ethical player model is beyond the scope of this paper, and will only be suggested when presenting the informational model of computer games as ethical experiences.

This paper is not an answer to effect studies research, nor it intends to clarify the possible ethical problems of violent games and children. ${ }^{5}$ This is a philosophy paper, and as such it contributes to the understanding of the ethics of computer games by means of an abstract model for the analysis of the moral relations between a player, and a designed system. The model illustrates how the values of the game system and the values of the player are deeply intertwined. These achievements can be used for further analysis of computer games ethics, both from a design, and a psychological or phenomenological theory.

This model will suggest a philosophical argument for understanding the ethical liability of computer games. While it is true that many games simulate violence, basing all their interactions in a glorification of gore, we need to understand their ontology, in order to qualify their ethical nature. In this paper I present an analytical tool that can be used to argue for games as generators of interesting ethical experiences - doing so by critically involving the player in the interaction system. In other words, many computer games require an ethical agent in order to create any type of ethical experience.

\section{Computer Games as Ethical Challenges}

Defcon can be a game of patience. Defcon gives the player command of a nuclear country. The goal is to eliminate as many enemy units as possible, while minimizing loses. In fact, to win the game, players have to lose the least. Defcon is a political simulator of atomic warfare, with a very clear message: in nuclear war, the winning condition is a losing condition.

\footnotetext{
${ }^{3}$ Floridi (2003a), (2003b).

${ }^{4}$ Floridi and Sanders (2003).

${ }^{5}$ Funk et al. (2004).
} 
Defcon is a multiplayer computer game played over the Internet. Players are given a limited number of units and resources that have to be distributed in the map. This first stage is a preparation for the nuclear showdown that inevitably will take place at the end of the round, when Defcon One is reached and players can use nuclear warheads. When the game reaches its end state, players will be rewarded with points according to the number of enemy units and civilians eliminated. The winner of the round is the player who loses the least, which implies that targeting large cities is the best strategy.

In the classic western theory of games, as exemplified by Huizinga ${ }^{6}$ and Caillois ${ }^{7}$, games are perceived as something "separate", as an "unproductive" activity that takes place within boundaries set by rules agreed upon by players ${ }^{8}$. Games are then arbitrary systems that establish constraints. Players have to accept these constraints in order to achieve the goals also proposed by the game system. Finally, games are considered entertainment, pastimes, vehicles for leisure with clear rules and unambiguous outcomes.

In more formal terms, games can be defined as activities in which practitioners interact with a system designed to create obstacles to the achievement of predetermined goals. To play a game is to give supreme but temporary importance to these constraints. For the player, nothing is more important than the rules and the goals of the game. Play is the focus of the self on artificial rules, worlds and goals. This focus brings forth ethical concerns, specially in the case of games played with computers.

In computer games, the rules are embodied in a virtual world. In many cases, the rules and the virtual world can be perceived by external observers as ethically harmless: there is no moral risk in New Super Mario Bros ${ }^{9}$ because the "violence" is cartoonish. But games like Defcon, or Doom, ${ }^{10}$ or Counter-Strike ${ }^{11}$ seem to create immediate ethical concern. These games could be understood, in a classic Virtue Ethics approach $^{12}$, as tools for the practice of the wrong virtues: violence, killing, inadequate conflict resolution. In this line of thought, a game has to be unethical because the agent is presented with rewards for actions of (simulated) evil, while feedback on the consequences is not provided 13 . The act of "killing" an opponent in a computer game becomes an action with no other consequence than what the game rules determine, therefore alienating the player from her reflections on the consequences of her actions.

This analysis of the ethics of games would then state that if players are faced with simulated evil and rewarded for their non virtuous actions in that gameworld, then players will internalize the lack of consequence for these actions. This process would be the first step in the desensitization effects that some researchers ${ }^{14}$ and media believe is the playing computer games.

In essence, this interpretation assumes that there is no difference between the act of launching an atomic bomb over Hiroshima and launching an atomic bomb over

6 See Huizinga (1950).

7 See Caillois (1958).

8 For a critical summary of the western understanding of the ontology of games, see Juul (2004), pp. 23-55.

9 Nintendo: 2006.

10 id Software: 1993.

11 Valve Software: 1999.

12 As defined by Aristotle's Nichomachean Ethics. See also Feezeel (2004).

${ }^{13}$ Nevertheless, it is necessary to say that the consequences of actions in games do have a system for feedback, embedded in the rule system, and usually tied to the winning conditions.

${ }^{14}$ See Carnagey et al. (2006), Funk et al. (2004). 
Tokyo in Defcon. Thus, playing computer games is an unethical act. This is both a limited understanding of computer games as cultural objects, and a very poor consideration of the ethical capacities of players as moral agents. Still, to counter argue, it is necessary to define what computer games are, and how players experience them. The rest of the paper will transform the alleged ethical shortcomings of computer games into analytical tools for defining computer games as ethical experiences. Computer games are not detached, encapsulated systems of meaning that classic game research has argued for. Computer games have a strong presence in the configuration of our ethical and cultural being, and as such we must describe them.

\section{Computer Games as Informational Systems}

When describing a game to someone that has never played it, the first stage is to describe the rules and the game mechanics. Players need to know what is allowed and possible, and what is not. Once players have a basic understanding of the rules, play is initiated. The initial states of play are approximations both to the rules and to the environment where we play. This process tries to find the winning strategies while staying true to the rules. Mastering a game is understanding the rules and mechanics of the game, how they interact with each other. Mastery leads to the behavioral patterns with which we play.

From a philosophical perspective, a game can be defined as an informational system: a construction of rules that determine which actions are meaningful or not within a certain experience, and how those actions can be performed. This ontology is based on the theory of Information Ethics. For Information Ethics, "the moral action itself can now be modeled as an information process, i.e., a series of messages (M), invoked by $a$, that brings about a transformation of states directly (...) affecting $p$, which may variously respond to $M$ with changes and/or other messages, depending on how $M$ is interpreted by $p$ 's methods" ${ }^{\prime 15}$. I will adapt this approach to encompass all kinds of ludic actions, defined as any action taken by an agent within a game system that is evaluated by a game rule. For example, placing the units in Defcon, firing a missile, or using the chat interface to communicate with other players, they all are ludic actions.

In order to understand the ethics of computer games from an informational perspective, it is relevant to define computer games within the terminology of Information Ethics. A computer game, then, is an infosphere, a "context constituted by the whole system of information objects, including all agents and patients, messages, their attributes and mutual relations" "16. In more classic computer game terms, a (game) infosphere is constituted by all game elements: players and AI agents, environments and gameworld, rules and game mechanics, and the interaction modes in the space of possibility ${ }^{17}$. In computer games there are agents, human or controlled by the game software. From a certain perspective, all agents operate similarly: interacting with the game state ${ }^{18}$ via game mechanics constrained by game rules. Playing a game is an act of agency within an infosphere, understanding agency as the interaction by means of exchange of information with a system

\footnotetext{
15 Floridi (2003a).

16 Floridi (2003), p. 8.

${ }^{17}$ Salen \& Zimmerman (2004), pp. 66-67.

${ }^{18}$ See Juul (2005), chapter 3.
} 
and other agents. ${ }^{19}$ This exchange of information is done via the methods ${ }^{20}$ of the different game objects, as constrained by the game rules.

In Defcon, players construct a strategy by carefully placing units in the places they are afforded by the rules, relative to their initial state in the game. Players manipulate the gameworld and their relations to other players in ways sanctioned and determined by the rules. Agency in Defcon, as in any computer game, is limited by the game rules and the mechanics afforded to players. The relation between players and the game environment will take place within the boundaries of those mechanics. The game as infosphere is determined by the design of the game.

From an ethical perspective, it is crucial to establish that the infosphere was designed to afford agency and allow for the flow of information in specific modes. Any system designed to modify or enhance agency, any system that is scripted ${ }^{21}$ for creating behaviors, has to raise ethical awareness. Altering agency can have ethical implications in the ontological status of the agents.

For instance, Defcon is designed to encourage conflict among players. All the mechanics and rules of the infosphere are geared towards creating conflict. Players of Defcon cannot find negotiated outcomes - the goal of the game is to simulate nuclear war and to reward those that annihilate more units of the opponent. The design of the game, as the design of any infosphere, is loaded with ethical values set to be experienced. More specifically, Defcon rewards those players who lose the least. Conventionally, wargames reward players who take out other players, regardless of their losses. Defcon modifies that rule, which leads to an ethical impact on the player experience.

The morals of game agents are not determined by the ethical values of the game design. Any agent in an infosphere, and particularly any human agent, has to be considered a moral agent. A moral agent is capable of ethically relating to the whole system, reflecting on her own values and ethics. Furthermore, moral agents are able of acting upon these values, modifying the actual ethics of the infosphere as experienced. While an infosphere can have ethical values imprinted in its design, it is the actual interaction of a moral agent with those ethical affordances and constraints ${ }^{22}$ what constitutes the ethics of a given infosphere. The informational ethics of a game system can only be analyzed when the game is experienced by agents, and not exclusively by its design, or its appearance.

Agents exert their ethical capacities by what Floridi has defined as creative stewardship. Human agents, from an Information Ethics perspective, ought to be considered homo poieticus, since they concentrate "not merely on the final result, but on the dynamic, ongoing process through which the result is achieved",23. Agents within an infosphere are not only in charge of exchanging information, but also of preserving the nature of the system, producing meaningful interactions. In computer games, this translates to the ethical responsibility for playing without cheating, not allowing other players to grief or harass the community, or developing and sharing interesting strategies for beating the game. Players

${ }^{19}$ See Floridi and Sanders (2004b) for a more detailed Information Ethics approach to the question of agency.

${ }^{20}$ Methods should be understood in the Object Oriented Programming paradigm, as the mechanisms an object has to access and/or manipulate data within other objects. See Weisfeld (2000).

21 See Latour (1992).

${ }^{22}$ See Norman (2002).

${ }^{23}$ See Floridi and Sanders (2005). 
as ethical agents are not mere input providers: they understand the nature of their actions within the semantics of the infosphere, and they act ethically.

This moral understanding in games is determined by two elements: the player as agent, and the cultural being that experiences play ${ }^{24}$. As players, we construct our agent values with those ethical affordances and constraints provided by the system. In Defcon, players' ethics reckon how the system encourages treason, since there is only one winner at the end of the game, but alliances with other players are encouraged. From an information ethics perspective, the player has a number of methods that allow her informational interaction with the other agents of the system. Those methods simulate nuclear war with the goal of devastating opponents and provoking casualties in their cities. The system is designed to encourage and reward that agency.

Players are not only input providers within the game system - their ethical configuration is also dependent on the ethics of the agent that becomes a player. The ethical agent outside of the gameworld is also relevant for understanding the ethics of computer games. Defcon is not only a state machine that simulates the conditions of conflict: it simulates nuclear war and its outcomes, representing them by means of an aural output system. Both the aural system and the simulation of nuclear war, what I will define as the semantic layer of the game, are interpreted by the human agents using their cultural and ethical knowledge.

For an analytical perspective, then, the infosphere comprises both the agents that interact with predetermined methods with the state machine, and the moral agents that evaluate the cultural and ethical relevance of in-game agency.

\section{The Method of Abstraction and the Ethics of Computer Games}

The Method of Abstraction ${ }^{25}$ provides a framework for analyzing the relation between agents and designed systems. In this paper I will use the concepts of Level of Abstraction (LoA henceforth) and Gradient of Abstraction (GoA henceforth). A Level of Abstraction is " finite but non-empty set of observables. No order is assigned to the observables" Gradient of Abstraction "is a formalism defined to facilitate discussion of discrete systems over a range of LoAs. Whilst a LoA formalises the scope or granularity of a single model, a GoA provides a way of varying the LoA in order to make observations at differing levels of abstraction" 27 . These two concepts, applied to computer games, allow the formulation of a model for the analysis of the ethics of computer games.

In computer games there are two dominant GoAs: one is limited to the direct interaction between agents and the state machine by means of game mechanics, the other comprises the game system as simulation and agents as ethical agents.

This first GoA defines all the input/output operations performed by and for the modification of the game state within the limitations of the rule system. For example, the action of selecting a unit in Defcon invokes a LoA in which the user interface, the class and particular instance of the unit, and the response from the state machine are relevant. I will define this GoA as the syntatic or procedural GoA. The syntatic/procedural GoA comprises the LoAs that regulate the input/output processes between agents and the

\footnotetext{
${ }^{24}$ See Gadamer (1975).

25 Floridi and Sanders (2004a).

${ }^{26}$ Floridi and Sanders: 2004a, p. 10.

27 ibid, p. 12.
} 
state machine. The syntactic/procedural GoA constitutes the inner mechanisms of the game, it's procedural nature 28 as a system.

The second GoA comprises the game system as simulation and agents as ethical agents. This expands the previous GoA by adding a semantic layer. The game system is in this layer more than a simple state machine: it comprises all the aural/aesthetic levels, giving cultural meaning to the elements of the game system. In Defcon, the state machine simulates nuclear war. The system behaviors, the semantic levels, its procedural and aesthetic content, they are all designed to be interpreted as war commanded from a nuclear bunker. This GoA comprises the procedural nature of the game with the cultural/aesthetic layers that wrap it, and it can be defined as a semantic/simulational GoA.

The agent in the semantic/simulational GoA is capable of reflecting morally not only about the appropriateness of her actions providing input, but also about the meaning of those actions. This meaning is interpreted both within the perspective of the cultural meaning of the simulation and with her own cultural values ${ }^{29}$.

The semantic GoA comprises the procedural GoA, and all the different LoAs present in a computer game can then be visualized within those relations. This conceptualization can be modeled in the next page.

At the heart of the ethical concerns with computer games lies the incapacity of understanding how players navigate in the infosphere. Common accusations against computer games understands them only within the procedural GoA, and thus from the perspective of an agent not concerned with anything else than providing the right input to modify the state machine. Furthermore, there seems to be a common issue to map the simulation within the procedural GoA, which is ontologically incorrect: the mechanical agent is not concerned with the simulation, it's beyond its LoA. When agents interact with the simulation, they use too their ethical capacities, since those are a part of their cultural resources required to understand how and why to play a game.

A common ethical concern raised by games is concerned with desensitization, which in this philosophical context I define as crisis of the agent's ethical tools for evaluating their conduct. ${ }^{30}$ This concern is based on the assumption that players allegedly provide input to the system without thinking about the consequences of their actions in the simulation, and thus becoming alienated from the causality reflection.

But given this Information Ethics analysis, this concern does not hold true. Any agent in a game operates within two distinct GoAs - one that cares about the procedural elements of the game, from user interface to score system, and another one that encapsulates that procedural part of the game into a larger GoA. In this larger GoA, the semantics of the game are extremely relevant, as they guide player interactions with the system.

${ }^{28}$ See Bogost (2006).

${ }^{29}$ This seems to discard any agent that is not human, but that is not the case: there are some LoAs in that GoA that can apply to agents that are not human: every LoA that does not require attention to those values external to the simulation can apply to artificial agents. A more detailed approach to artificial agency in infospheres can be found here: See Floridi and Sanders (2004b).

30 As opposed to the psychological approach, concerned with the reduction of emotional response: see Funk et al. (2004). A possible continuation of this paper would lead to comparative work in social psychology and philosophy, on the ethical and emotional effects of these games in different ethical agents, mapped to the informational structure of the game, as modeled in this paper. 


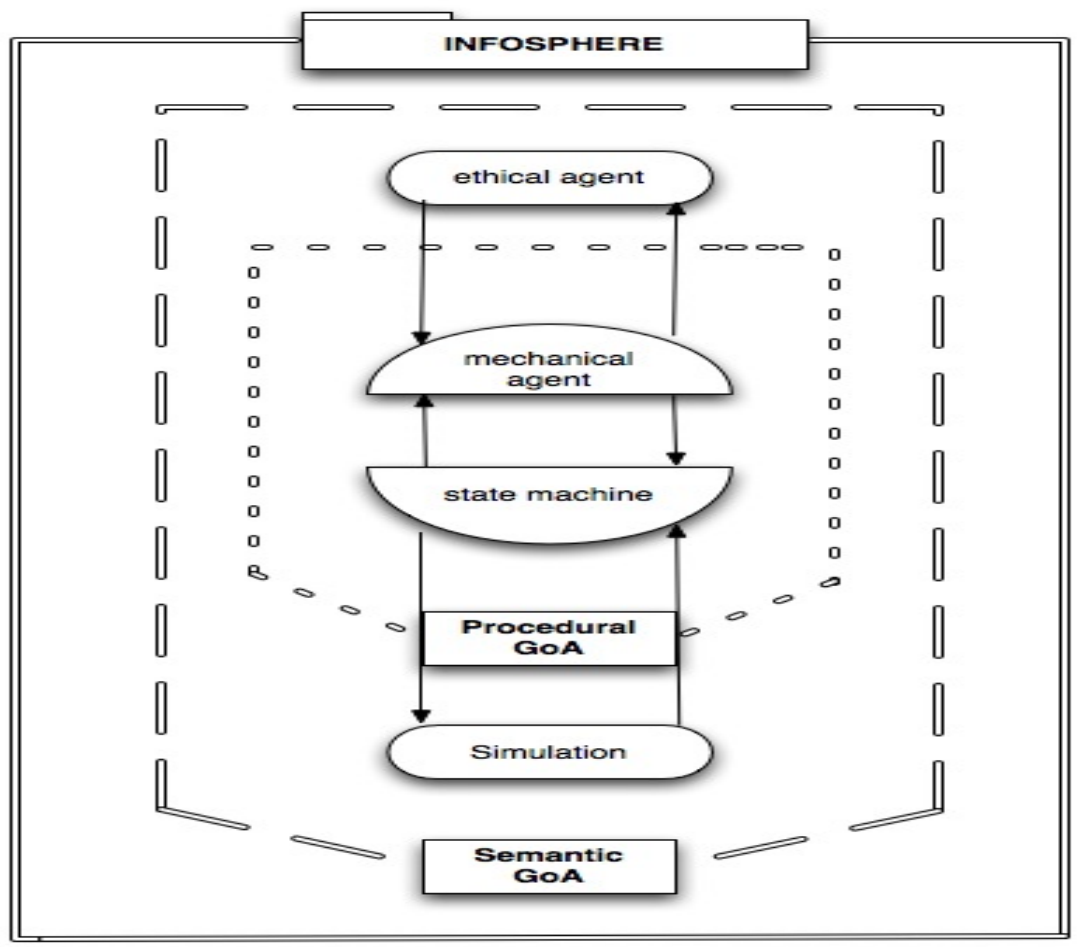

This process can be understood with an analogy: piano players often need to "warm" their fingers before playing. They do so by playing on the piano a number of exercises. Piano players are not concerned with the semantic elements of the music piece, only with the mechanical interaction with the piano. Once they start playing a piece, though, the semantic layer is also present, so tone, emotion, phrasing, and the aesthetic qualities of playing piano require attention.

This informational perspective on the ontology of games provides an framework for the analysis of the ethics of computer games. It also gives arguments as to why some computer games are not relevant for ethical theory. Ethically irrelevant games are those in which the procedural dominates over the semantic. For instance, in Tetris $^{31}$ players don't need to understand the simulation in order to successfully interact with the system. To play Tetris is enough with an understanding of the rules and mechanics. Abstract games ${ }^{32}$, then, are those games that privilege the importance of the procedural over the semantic GoA.

Summarizing, the procedural GoA comprises the design and implementation of the game as a state machine, with the basic mechanics and rules that determine the interaction of input agents with the system. The semantic GoA comprises the layers of meaning that we understand as the gameworld - the reasons why players are emotionally attached to the game, understand how to play it, and take choices. Agents in this gradient are concerned with the player community, the cultural and ethical values of

${ }^{31}$ Alexei Pajitnov, 1985.

32 See Juul (2005), pp. 130-131. 
the game, and the connections of the game with larger infospheres external to the game experience.

The games that taunt players with ethical decision-making, like Fable or Knights $^{33}$ of the Old Republic ${ }^{34}$ are flawed because their alleged ethical thinking is placed in the procedural layer: "evil" is not understood as a dominant semantic condition but a procedural one, as a state in the machine. Ethical agents do not require to use their ethical reasoning within the semantic layer in order to take a choice: it is enough to understand the arbitrary ethics assigned to a particular game state, and let the game system evaluate the behavior. These games generate a process of desensitizing the agent of their ethical thinking about the simulation. Since ethics are limited to the procedural layer, agents are deprived of their ethical capacities in favor of an external system that will evaluate their choices.

\section{Conclusions}

This paper has presented an analytical framework of the ethics of computer games as informational environments. This framework is based on Information Ethics, and provides a comprehensive approach to the technological and cultural challenges of computer games' ethics.

The model I have presented in this paper can be used not only to analyze the ontology of games as ethical systems, but also to provide a philosophical answer to issues concerning games and unethical content. That argument goes as follows: any computer game requires a player who understands not only the basic rules and interactions, but also the meaning of the game world, of the simulation. A player that can understand that sematic layer is an ethical agent, and as such will always use moral maturity to interpret and experience the game. In other words - players are ethical agents that require ethical thinking to interact with the game.

A subject for further research is the meaning of ethical thinking for players. I would argue that ethics, like Aristotle wrote, is a practical science that requires maturity, a constant practice towards perfection. Similarly, the ethical maturity of players evolves with time and experience of games, and thus social instances like age regulation codes, are effective tools for guaranteeing the successful development of players as ethical agents.

Computer games offer experiences of seemingly impossible worlds that put players in the center of epic tales of heroism. But the moral dimension of games is still in its infancy. With this paper I have introduced a framework for understanding games as ethical systems. The next step is to put into practice the lessons learnt from this reflection, since the possibility of creating engaging ethical gameplay is the true promise of any next generation gaming.

\section{References: Literature}

Bateman, C., Boon, R.: 21st Century Game Design. Charles River Media, Massachusetts (2005)

Bogost, I.: Persuasive Games. The Expressive Power of Videogames. The MIT Press, Massachusetts (2006)

\footnotetext{
33 Lionhead: 2004.

34 Bioware: 2003.
} 
Caillois, R.: Man, Play and Games. Translated by Meyer Barash. University of Illinois Press, Urbana (1958/2001)

Carnagey, N.L., Anderson, C.A., Bushman, B.J.: The effect of video game violence on physiological desensitization to real-life violence. Journal of Experimental Psychology (2006)

Feezell, R.: Sport, play, and ethical reflection. University of Illinois Press, Urbana (2004)

Floridi, L., Sanders, J.: Internet Ethics: the Constructionist Values of Homo Poieticus. In: Cavalier, R. (ed.) The Impact of the Internet on Our Moral Lives, New York, SUNY (2005)

Floridi, L., Sanders, J.: Levellism and the Method of Abstraction, Information Ethics Group Research Report (2004a),

http: / / www.philosophyofinformation.net/pdf / latmoa.pdf

Floridi, L., Sanders, J.: On the Morality of Artificial Agents. Minds and Machines 14(3), 349$379(2004 b)$

Floridi, L.: On the Intrinsic Value of Information Objects and the Infosphere. Ethics and Information Technology 4(4), 287-304 (2003a)

Floridi, L.: Two Approaches to the Philosophy of Information. Minds and Machines 13(4), 459-469 (2003b)

Funk, J.B., Baldacci, B., Heidi, Pasold, Tracie, Baumgardner, Jennifer: Violence exposure in real-life, video games, television, movies, and the internet: is there desensitization? Journal of Adolescence 27, 23-29 (2004)

Gadamer, H.G.: Truth and Method (1975); Translated by Weinsheimer, J., Marshall, D.G. Continuum, New York (2004)

Huizinga, J.: Homo Ludens. The Beacon Press, Boston (1950)

Juul, J.: A Certain Level of Abstraction. In: Proceedings of the III DiGRA Conference (2007)

Juul, J.: Half-Real. Video Games between Real Rules and Fictional Worlds. The MIT Press, Massachusetts (2005)

Latour, B.: Where are the Missing Masses? - the Sociology of a Few Mundane Artifacts. In: Bijker, W., Law, J. (eds.) Shaping Technology/Building Society, pp. 225-258. The MIT Press, Massachusetts

Norman, D.: The Design of Everyday Things. Perseus, New York (2002)

Salen, Katie, Zimmerman, Eric: Rules of Play. Game Design Fundamentals. The MIT Press, Massachusetts (2004)

Suttton-Smith, B.: The Ambiguity of Play. Harvard University Press, Cambridge (1997)

Weisfeld, M.: The Object Oriented Thought Process. Sams Publishing, Indianapolis (2000)

\section{References: Games}

Bioware (2003), Knights of the Old Republic

Blizzard Entertainment (2005), World of Warcraft

CCP Games (2003), Eve Online

id Software (1993), Doom

Io Storm (2000), Deus Ex

Lionhead Studios (2004), Fable

Newsgaming.com (2004), September 12th

Nintendo (2006), New Super Mario Bros

Pajitnov, A. (1985), Tetris

Quantic Dream (2005), Fahrenheit

Rockstar Games (2003), Manhunt

Sony (2005), Shadow of the Colossus

Valve Software (1999), Counter-Strike 